\title{
Vesícula biliar multiseptada. Una anomalía congénita infrecuente
}

Drs. Nelson Menocal $F^{(1)}$, Aurora Garrote $P^{(1,2)}$, Diana García $C^{(1)}$, José Miguel Alonso $S^{(3)}$, José Angel Santos $\boldsymbol{S}^{(3)}$.

1. Médico Interno Residente de Radiodiagnóstico. Hospital Universitario de Salamanca. España.

2. Especialista en Medicina Familiar y Comunitaria.

3. Especialista en Radiodiagnóstico. Hospital Universitario de Salamanca. España.

\section{Multiseptate gallbladder. An unusual congenital anomaly}

\begin{abstract}
Multiseptate gallbladder is an extremely rare congenital anomaly characterized by the presence of multiple septa that divide the lumen of the gallbladder. Although some patients are asymptomatic and the anomaly is found incidentally, the most frequent clinical presentation is chronic pain in the right upper quadrant. Ultrasound is the imaging technique of choice for diagnosis, and cholangioMR is used mainly to rule out associated anomalies of the biliary tract. In this article we report the case of a patient with right upper quadrant pain of several years of evolution, where imaging through abdominal ultrasound and MRI allowed a confident diagnosis.
\end{abstract}

Keywords: Multiseptate gallbladder, Gallbladder anomalies, Magnetic resonance imaging, Ultrasonography.

Resumen: La vesícula biliar multiseptada es una anomalía congénita extremadamente rara, que se caracteriza por la presencia de múltiples septos que dividen la luz de la vesícula biliar. La forma más frecuente de presentación clínica es el dolor crónico en el cuadrante superior derecho, aunque algunos pacientes permanecen asintomáticos y se descubre de manera casual. La técnica de imagen de elección para su diagnóstico es la ecografía y se reserva la resonancia magnética y colangio-resonancia magnética para descartar anomalías asociadas de la vía biliar. Presentamos el caso de una paciente con dolor en hipocondrio derecho de varios años de evolución, a la que se le realizó una ecografía abdominal y posteriormente una resonancia magnética, identificando en ambas pruebas de imagen una vesícula biliar con múltiples septos finos dividiendo su luz.

Palabras clave: Resonancia magnética, Ultrasonografía, Vesícula biliar, Vesícula biliar multiseptada.

Menocal N y cols. Vesícula biliar multiseptada. Una anomalía congénita infrecuente. Rev Chil Radiol 2011; 17(4): 176-178.

Correspondencia: Dr. Nelson D. Menocal P. / nmenocal@yahoo.es

Trabajo recibido el 08 de mayo de 2011, aceptado para publicación el 24 de junio de 2011.

\section{Introducción}

La vesícula multiseptada forma parte de las anomalías congénitas de la vesícula biliar. Es una entidad extremadamente infrecuente que suele diagnosticarse en niños y adultos jóvenes con dolor en hipocondrio derecho. A veces no provoca síntomas y se descubre de manera incidental en pacientes de mayor edad al realizar una ecografía abdominal por otro motivo ${ }^{(1,2)}$.

Desde su primera descripción en la literatura radiológica por Simon y Tandon en 1963, se han publicado menos de cincuenta $\operatorname{casos}^{(1,3)}$.

Suele ser una entidad que se presenta aislada o asociada a otras anomalías de la vía biliar como hipoplasia vesicular, quistes de colédoco, ectopia vesicular y anomalías de la unión bibliopancreática ${ }^{(3,4)}$. Aunque su curso clínico puede ser indolente, se han descrito cuadros de colecistitis y cólicos biliares.

Estudios recientes sugieren la utilización de ecografía y resonancia magnética $(\mathrm{RM})$ para su diagnóstico e identificación de anomalías biliares asociadas.

Presentamos un caso de vesícula multiseptada diagnosticada por medio de ecografía y RM.

\section{Presentación del caso}

Paciente mujer de 22 años de edad, sin antecedentes personales de interés, remitida al servicio de 
urgencias de nuestro hospital por dolor abdominal de predominio en cuadrante superior derecho de varios años de evolución, que en los últimos días ha aumentado de intensidad. En la exploración física presentaba dolor localizado en hipocondrio y flanco derecho, con signo de Murphy dudoso. Las pruebas de laboratorio no presentaban alteraciones.

Se realizó ecografía abdominal que demostró una vesícula biliar de tamaño normal, discretamente lobulada, sin colelitiasis, de grosor parietal dentro de límites normales y con múltiples tabiques finos ecogénicos, intraluminales que ocasionaban una apariencia en panal (Figura 1). No se visualizó dilatación de la vía biliar intra ni extrahepática.

Posteriormente, se realizó RM que confirmó los hallazgos ecográficos y permitió excluir otras anomalías biliares (Figura 2).

La paciente fue dada de alta con tratamiento analgésico y actualmente está en seguimiento por el Servicio de Aparato Digestivo.
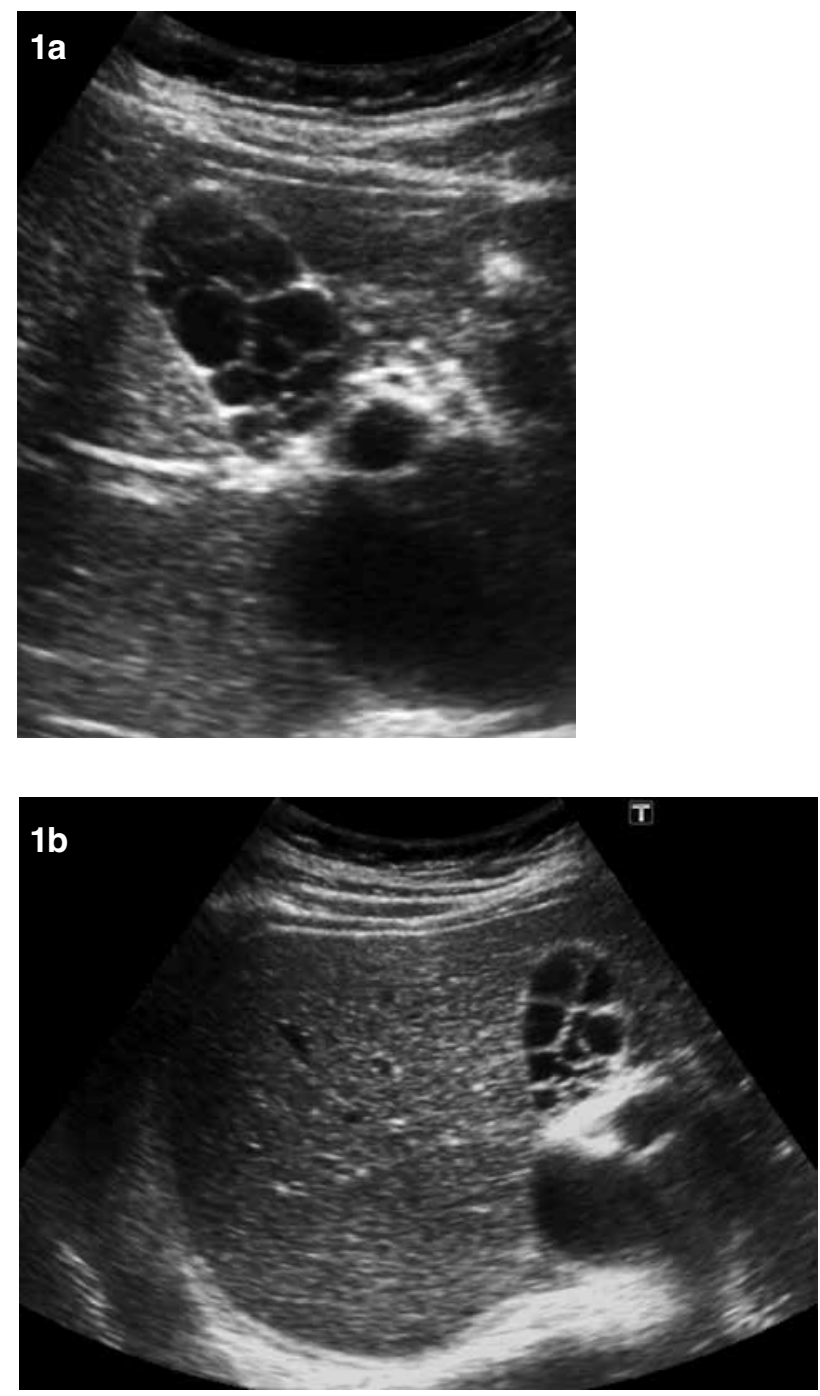

Figura 1. (a y b) Ecografía de hipocondrio derecho, en la que se observa una vesícula biliar multiseptada con aspecto multilocular en "panal de abeja".
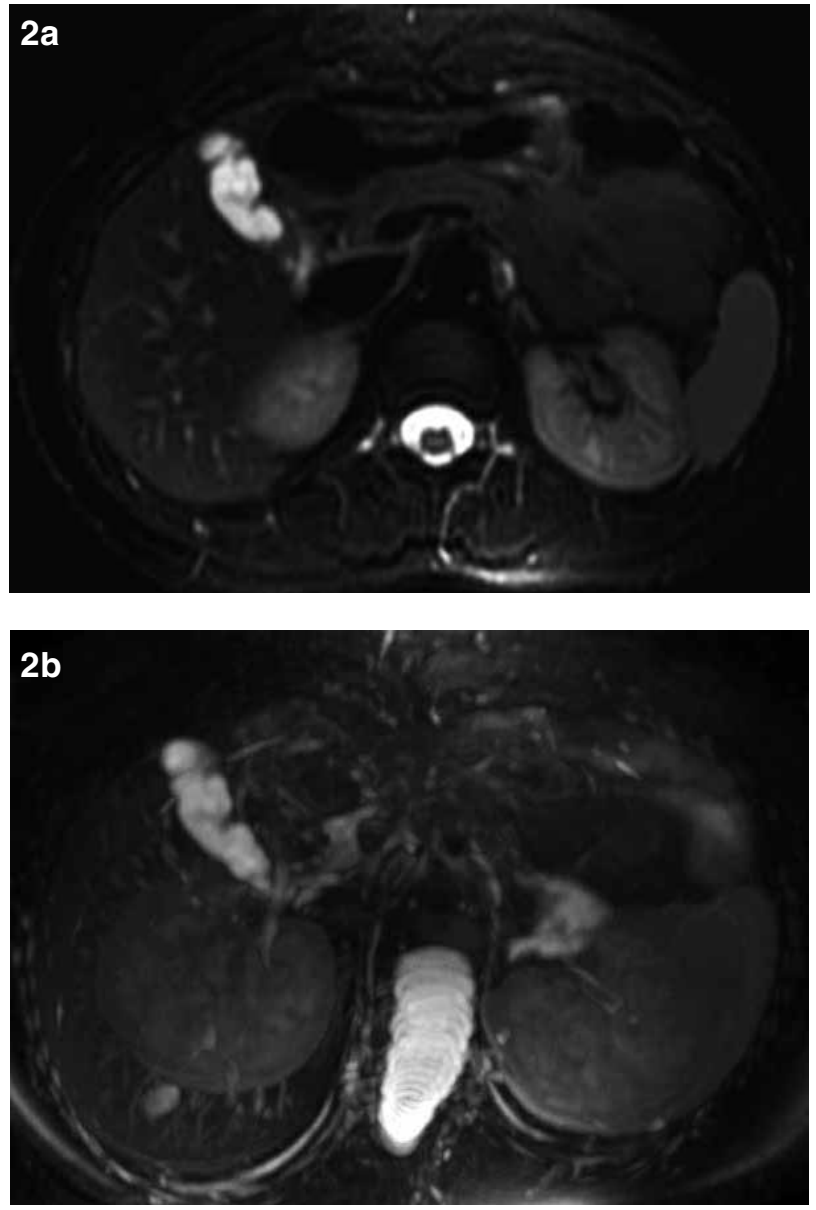

Figura 2. (a y b) RM (potenciada en T2) y reconstrucción MPR coronal modificada donde se visualiza vesícula biliar lobulada, con septos hipointensos en su interior.

\section{Discusión}

Las anomalías congénitas de la vesícula biliar han sido clasificadas en: anomalías de forma, número, localización, tamaño y de ausencia. La vesícula biliar multiseptada es una entidad extremadamente rara, incluida dentro de las anomalías de forma ${ }^{(1,4,5)}$.

El mecanismo embriológico exacto por el cual se desarrolla esta alteración no está bien establecido. Teniendo en cuenta que la luz de la vesícula biliar se forma por la coalescencia de cavidades intraepiteliales en la yema vesicular, una vacuolización incompleta podría ser la responsable de la formación de septos ${ }^{(1-3,6,7)}$.

Clínicamente los pacientes pueden encontrarse asintomáticos o presentar dolor crónico en hipocondrio derecho o en epigastrio, cólicos biliares, colecistitis o incluso pancreatitis. Estos síntomas probablemente estén en relación con alteraciones en el vaciamiento de la vesícula, bien por dificultad de excreción de la bilis o a problemas de contracción de la vesícula, que secundariamente producen un aumento de la presión intraluminal ${ }^{(2,3,8)}$. A pesar del éstasis, la ictericia es poco frecuente. En nuestro caso la paciente solamente 
presentaba dolor crónico de años de evolución, sin otra sintomatología.

Se han usado varias técnicas de imagen para realizar el diagnóstico de esta entidad, entre ellas: colecistografía oral e intravenosa, ecografía, tomografía computarizada o RM. Otras modalidades incluyen el uso del tecnecio $99 \mathrm{~m}^{(7)}$. Independientemente de la técnica de imagen utilizada los hallazgos radiológicos son similares, observando una vesícula de tamaño normal, de contornos lobulados, dividida por múltiples septos o tabiques internos que forman cavidades quísticas, dando un aspecto multiloculado en "panal de abeja" $(1,3,7,9,10)$. Muchos autores han descrito las ventajas e inconvenientes de cada una de las técnicas de imagen, aunque hoy en día se considera que la ecografía es la técnica de elección por su bajo coste, alta disponibilidad y ausencia de radiaciones, reservando la colangio-RM para descartar anomalías asociadas de la vía biliar.

A pesar de ser una entidad infrecuente, la vesícula multiseptada no suele presentar problemas de diagnóstico. La adenomiomatosis, la colesterolosis, la colecistitis necrotizante y el quiste hidatídico pueden crear confusión en algunas ocasiones ${ }^{(3,9,10)}$.

Aunque la ecografía y la RM en la mayoría de las ocasiones son pruebas suficientes para su diagnóstico, la confirmación definitiva se obtiene mediante análisis patológico de la pieza de colecistectomía. La vesícula se encuentra dividida en múltiples compartimientos por septos formados por epitelio columnar, tejido conectivo y fibras de músculo liso. La presencia de tejido muscular liso confirma el origen congénito ${ }^{(1,3,10)}$.

En los pacientes asintomáticos no es preciso realizar ningún tratamiento quirúrgico, mientras que en los casos sintomáticos está indicada la colecistectomía, preferiblemente vía laparoscópica ${ }^{(1,10)}$.

En conclusión, la vesícula multiseptada es una entidad muy rara, generalmente asintomática, pero que debe considerarse en el diagnóstico diferencial de aquellos pacientes con dolor crónico en hipocondrio derecho y con una vesícula de aspecto multiloculado en "panal de abeja". La ecografía es el estudio de elección y se reserva la RM o la colangio RM para descartar anomalías biliares asociadas.

\section{Bibliografía}

1. García E, Herraiz I, Burgueño FJ. Vesícula multiseptada. Radiología 2009; 51: 532-533.

2. Bhagaban BS, Amin PB, Land AS, Weinberg T. Multiseptate gallbladder. Embryogenetic hypotheses. Arch Pathol 1970; 89: 382-385.

3. Pérez R, Hervás V. Vesícula multiseptada. Radiología Abdominal 2010; 7: 16.

4. Yamamoto T, Matsumoto J, Hashiguchi Sh, Yamaguchi A, Sakoda K, Taki Ch. Multiseptate gallbladder with anomalous pancreaticobiliary ductal union: A case report. World J Gastroenterol 2005; 11: 6066-6068.

5. Patel NR, Joshipura VP, Haribhakti SP, Soni HN. Septate gallbladder in the laparoscopic era. J Minim Access Surg 2008; 4: 20-22.

6. Lev-Toaff A, Friedman A, Rindsberg S, Caroline D, Maurer A, Radecki P. Multiseptate Gallbladder: Incidental Diagnosis on Sonography. AJR 1987; 148: 1119-1120.

7. Nakazawa T, Ohara H, Sano H, Kobayashi S, Nomura $\mathrm{T}$, Joh T, "et al." Multiseptate gallbladder: Diagnostic value of MR cholangiography and ultrasonography. Abdom Imaging 2004; 29: 691-693.

8. Simon M, Tandon BN. Multiseptate gallbladder: a case report. Radiology 1963; 80: 84-86.

9. Demirpolat M, Duygulu G, Tamsel D. Multiseptate gallbladder in a child with recurrent abdominal pain. Diagn Interv Radiol 2010; 16: 306-307.

10. Kocakoc E, Kiris A, Alkan A, Bozgeyik Z, Sen Y, Ozdemir $\mathrm{H}$. Multiseptate gallbladder in a child with chronic abdominal pain: ultrasonography, magnetic resonance imaging and magnetic resonance cholangiography findings. European Journal of Radiology Extra 2003; 47: $22-25$. 